\section{Novel 3D in vitro retina model to investigate mechanisms involved in age-related macular degeneration}

\section{Beatrice Belgio, Sara Mantero, Federica Boschetti}

Department of Chemistry, Materials, and Chemical Engineering "Giulio Natta”, Politecnico di Milano, Milano, Italy

\begin{abstract}
In the present study, we present a strategy to develop a 3R-compliant in vitro retina model to study age-related macular degeneration pathways.
\end{abstract}

\section{Introduction}

Age-related macular degeneration (AMD) is one of the leading causes of irreversible vision loss worldwide, affecting globally 170 million people. ${ }^{1}$ AMD consists in a progressive degeneration of the retinal pigment epithelium (RPE) and the underlying Bruch's membrane (BrM). Current treatments are unable to reverse vision damage. This is related to incomplete knowledge of the basic pathological mechanisms involved. So far, animal models have been employed to investigate AMD pathways. However, high-scale in vivo methods are complex to establish. In addition, the $3 \mathrm{R}$ principle has urged to use alternative approaches. Hence, in the present study, we aimed to develop a suitable 3R-compliant three-dimensional (3D) in vitro model, including a BrM model and the RPE layer. To build an in vitro model that mimics the native tissue, we investigated the biomechanics of native porcine retina. In line with the $3 \mathrm{R}$ principle, pig eyes were collected from a local abattoir applying the reuse of animal, thus reducing the number of animals used for research purposes.

The in vitro model will be crucial to study and acquire greater insight into the pathological mechanisms involved and to develop and test new therapies, which cure AMD.

\section{Materials and Methods}

BrM model was fabricated with the EF300 electrospinning system (SKE research equipment, Leonardino s.r.l.) using a $15 \%(\mathrm{wt} / \mathrm{v})$ polymer solution, composed of polycaprolactone (PCL, Sigma) and Bombyx mori Silk Fibroin (SF) (weight ratio 95:5 $\mathrm{PCL} / \mathrm{SF}$ ).

$\mathrm{BrM}$ model was characterized in terms of morphology, mechanical properties, permeability, and biocompatibility. Morphological analyses were performed using scanning electron microscopy (SEM, Stereoscan 360, Cambridge Instruments) at $10 \mathrm{kV}$ and Image J software (National Institute of Health). A mechanical testing machine (Synergie 200, MTS Systems) equipped with a $100 \mathrm{~N}$ loading cell was used for tensile test. Samples were first preconditioned and then pulled to failure at 0.1 $\mathrm{mm} / \mathrm{s}$. The same protocol was applied to hydrated samples, i.e. samples submerged in saline solution for 1 hour. Stiffness was calculated by measuring the slope of the initial linear region of the resulting stressstrain curve. To estimate permeability, a custom-made apparatus was employed. Constant hydrostatic pressures were applied to the sample and the flow rate was measured to calculate Darcy's permeability and then hydraulic conductivity. To investigate the in vitro biocompatibility of the BrM model, a direct contact cytotoxicity test was carried out for 5 days using SEM. $4 \times 10^{5}$ ARPE-19 cells (ATCC) were seeded on the BrM electrospun model. For retinal mechanical measurements, 9-month-old pig eyes were collected within 1 hour postmortem. Retinal samples were isolated and pulled until failure at $0.1 \mathrm{~mm} / \mathrm{s}$ by using an electromechanical testing machine (Bose EnduraTEC ELF 3200, Eden Prairie) equipped with a $22 \mathrm{~N}$ load cell. The elastic modulus (E) was extrapolated from the stress-strain curves as the linear portion of the curve before the change of the slope.

Results are presented as mean \pm Standard Deviation (SD). Differences were considered statistically significant if the $p$ value was less than 0.05 .

\section{Results}

The fabricated BrM model presented a randomly oriented fibrous network with an average fibre diameter of $1217 \pm 101 \mathrm{~nm}$ and a thickness of $44 \mu \mathrm{m}$. The mechanical properties of the BrM model were reflected by a typical tensile stress-strain curve. No
Correspondence: Beatrice Belgio, Department of Chemistry, Materials, and Chemical Engineering "Giulio Natta", Politecnico di Milano, 20133 Milano, Italy.

E-mail: beatrice.belgio@polimi.it

Key words: Retina; in vitro model; 3R; agerelated macular degeneration; biomechanics.

Acknowledgments: The authors acknowledge Fumagalli Industria Alimentari, Dr Arpa, and Leonardino SRL.

Disclosures: Authors have nothing to disclose. Conference presentation: This paper was presented at the Third Centro 3R Annual Meeting - L'era delle 3R: modelli in silico, in vitro e in vivo per promuovere la ricerca traslazionale 30 September - 1 October 2021, Evento online organizzato dal Politecnico di Torino.

Received for publication: 9 July 2021.

Accepted for publication: 7 September 2021.

This work is licensed under a Creative Commons Attribution NonCommercial 4.0 License (CC BY-NC 4.0).

CC Copyright: the Author(s), 2021

Licensee PAGEPress, Italy

Biomedical Science and Engineering 2021; 4(s1): doi:10.4081/bse.2021.150

statistically significant difference was found between dry and hydrated test conditions. The average values of stiffness and hydraulic conductivity of the BrM model were in agreement with those estimated for native BrM (Table 1).2,3 Finally, BrM model proved to be biocompatible, as cells well adhered and spread on its surface (Figure 1). Based on BrM biocompatibility, RPE layer will next be bioprinted on the BrM

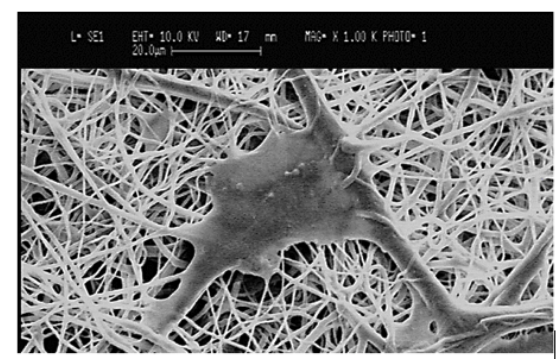

Figure 1. SEM image of an ARPE-19 cell on the BrM model.

Table 1. Stiffness and hydraulic conductivity of the BrM model and native BrM.

\begin{tabular}{lcc} 
Parameter & Native BrM & BrM model \\
Stiffness [MPa] & $7-14[2]$ & $13 \pm 3.6$ \\
Hydraulic conductivity [m/(Pas)] & From 20 to $100 \times 10^{-10}[3]$ & $20 \times 10^{-10}$ \\
\hline
\end{tabular}


model. To develop a suitable bioink, a material with appropriate mechanical properties has to be selected. However, retinal mechanical properties have been poorly characterized. Therefore, we carried out tensile tests on porcine retina. The resulting stress-strain curve of retinal samples under tensile tests is shown in Figure 2. The curve exhibited a narrow initial linear region followed by a wide region of plastic behavior

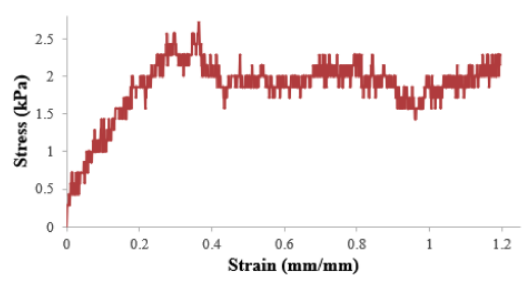

Figure 2. Stress-strain plot of porcine retina under tensile test. presenting several peaks probably due to micro-ruptures in the tissue. The average value of $\mathrm{E}$ was $13.4 \pm 6.7 \mathrm{kPa}$. This outcome will help us to build an in vitro retina model that better replicates the native tissue.

\section{Discussion and Conclusions}

In this study, novel BrM-mimetic substrates were fabricated as part of an in vitro retina model. The substrates displayed structural and mechanical similarity to physiological BrM. Moreover, we proved that the BrM model can be used as a substrate for RPE layer. To better reproduce RPE layer, we characterized retinal biomechanics with a tensile test. Thanks to this outcome, we will develop a suitable bioink for the 3D Bioprinting of RPE on the BrM model, thus obtaining a $3 \mathrm{D}$ in vitro retina model. The model will be used to study
AMD progression and develop new effective therapies, thus reducing the number of animal experiments.

\section{References}

1. Wong WL, Su X, Li X, et al. Global prevalence of age-related macular degeneration and disease burden projection for 2020 and 2040: a systematic review and meta-analysis. Lancet Glob Health 2014;2:e106-16.

2. Ugarte M, Hussain AA, Marshall J. An experimental study of the elastic properties of the human Bruch's membranechoroid complex: relevance to ageing. Br J Ophthalmol 2006;90:621-6.

3. Moore DJ, Clover GM. The Effect of Age on the Macromolecular Permeability of Human Bruch's Membrane. Invest. Ophthalmol. Vis Sci 2001;42:2970-5. 